\title{
Influence of Temperature on Magnetostrictive Delay Line Properties
}

\author{
J. SAlACH ${ }^{a, *}$, T. CharUbin ${ }^{a}$, P. NOWAK ${ }^{b}$ AND D. JACKIEWICZ ${ }^{b}$ \\ ${ }^{a}$ Institute of Metrology and Biomedical Engineering, Faculty of Mechatronics, Warsaw University of Technology, \\ sw. A. Boboli 8, 02-525 Warsaw, Poland \\ ${ }^{b}$ Industrial Research Institute for Automation and Measurements PIAP, \\ Al. Jerozolimskie 202, 02-486 Warsaw, Poland
}

\begin{abstract}
This paper presents results of tests performed on magnetostrictive delay line concerning its change in properties during temperature changes. Results indicate that an increase in temperature causes a decrease in wave amplitude and increase in the speed of propagation. The material used for the magnetostrictive delay line was an amorphous steel alloy of $\mathrm{Fe}-\mathrm{Si}-\mathrm{B}$ in tape form.
\end{abstract}

DOI: 10.12693/APhysPolA.131.1183

PACS/topics: $75.80 .+\mathrm{q}, 75.50 . \mathrm{Kj}, 81.70 .-\mathrm{q}$

\section{Introduction}

Delays are commonly used in displacement sensors where other type of sensors cannot be used, for example, liquid level or position sensors of a working head [1]. On the other hand, the use of amorphous tape in magnetostrictive delay line is a new idea. This type of tapes are widely used in electrical components, as well as in force and stress sensors [2], but their use in the delay lines is not yet properly researched. This type of device has however also been used for measurements of stresses and forces in the ceramic components [3]. Therefore, it is very important that these sensors have high sensitivity and are resistant to the influence of external factors, such as temperature. As part of the work a test to study the influence of temperature on magnetostrictive delay lines was performed and the measurements were carried out. The research concerned the effect of change in temperature on the performance parameters of magnetostrictive delay line in the range from $25^{\circ} \mathrm{C}$ to $70^{\circ} \mathrm{C}$. The results show a slight influence of temperature on both the amplitude and speed of propagating wave in the delay line.

\section{Methodology of investigation}

The magnetostrictive material used in the delay line was an amorphous alloy Metglas 2605S3A [4]. It is an iron-based amorphous alloy, with about $12 \%$ boron and about $9 \%$ silicon content. The width of the tape was $8 \mathrm{~mm}$ and the thickness was $25 \mu \mathrm{m}$. Elastic modulus of the material is $100-110 \mathrm{GPa}$, coercivity $-<8 \mathrm{~A} / \mathrm{m}, \mathrm{DC}$ permeability $\longrightarrow>20000$, and saturation magnetostriction $-20 \mathrm{ppm}$.

*corresponding author; e-mail: j.salach@mchtr.pw.edu.pl
The most important parameter from the point of view of magnetostrictive delay line is saturation magnetostriction of the material, because it is responsible for the efficiency of the propagating signal in the delay line. For the tested material it is equal to $20 \mathrm{ppm}$. Another important parameter is the modulus of elasticity. It is responsible for the propagation velocity of the wave in the magnetostrictive material and is dependent on the ambient temperature. For the test material it is in the range of 100-110 GPa. The stand used in the measurement process consisted of the magnetostrictive delay line inserted into a furnace. The furnace ensured stable temperatures throughout the experiment. During tests the amorphous tape was subjected to a $50 \mathrm{~g}$ stress placed on one end. The tape measured $900 \mathrm{~mm}$ in length, the distance between coils was $550 \mathrm{~mm}$. Figure 1 presents a draft of the measurement stand.

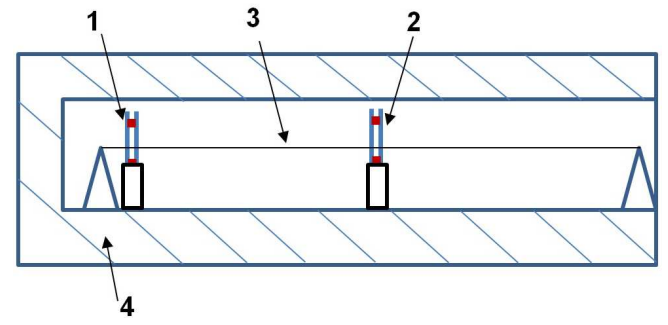

Fig. 1. Schematic of measurement stand: 1 - transmitting coil, 2 - receiving coil, 3 - amorphous magnetostrictive tape, 4 - furnace, 5 - weight tensioning tape.

The wave propagating through the line was induced by a single square edge applied to coil 1 and was received by coil 2 .

In order to remove quantization noises the results were filtered in Octave software using third-order digital Butterworth filter with sampling frequency to cut-off frequency ratio of 25. Using Butterworth filter assured min- 
imal amplitude distortion. The measuring stand assured stable test temperatures in the range of $25^{\circ} \mathrm{C}$ to $70^{\circ} \mathrm{C}$, with the uncertainty of measurement of $\pm 1^{\circ} \mathrm{C}$. Higher temperatures were not achievable due to the mechanical components made of plastic material.

\section{Results}

Figures 2 and 3 present measured waveforms for different temperatures.

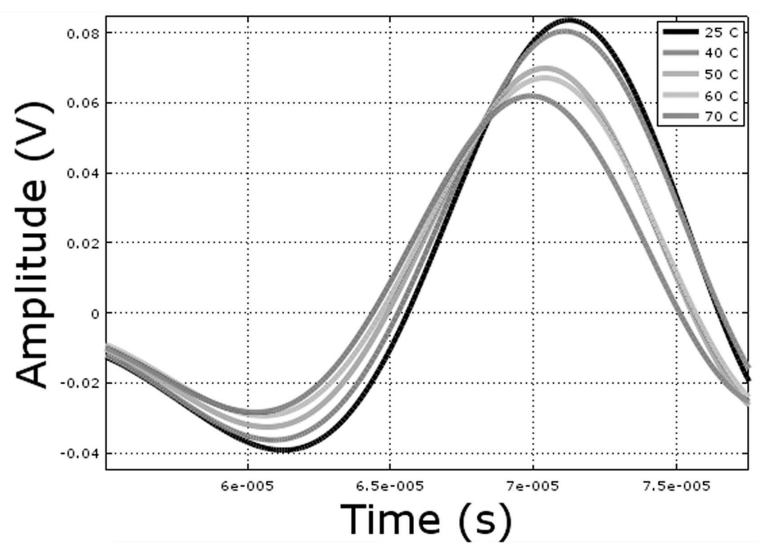

Fig. 2. Receiving coil charts for different temperatures (1 period).

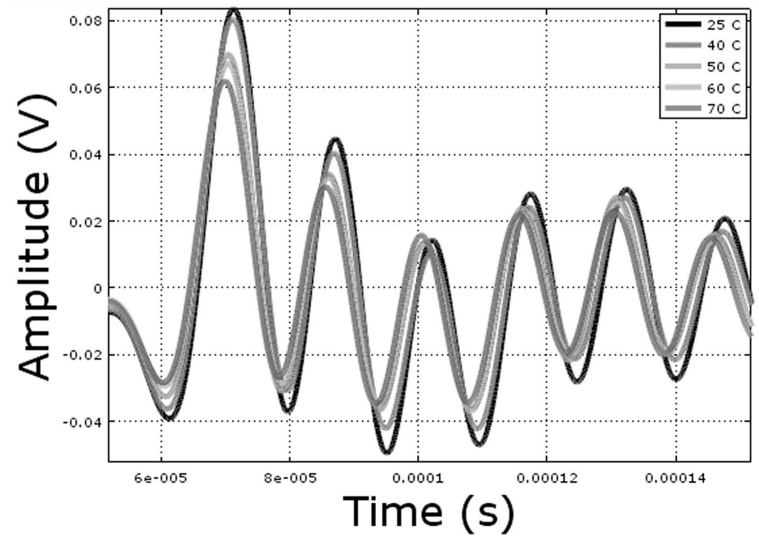

Fig. 3. Receiving coil charts for different temperatures (6 periods).

Table II presents amplitude and time delay measurements in function of temperature.

Increase in operating temperature caused relatively large changes in amplitude and small changes in time delay of received signals. The main cause of these is the Young (elastic) modulus dependence on the temperature of the steel tape. Figures 4 and 5 present relative amplitude and delay errors in function of temperature.
TABLE I

Peak to peak amplitude and delay changes in function of temperature.

\begin{tabular}{c|c|c}
\hline \hline Temperature $\left[{ }^{\circ} \mathrm{C}\right]$ & Amplitude $\left[\mathrm{V}_{p-p}\right]$ & Delay $[\mu \mathrm{s}]$ \\
\hline 25 & 0.133 & 65.6 \\
40 & 0.123 & 65.3 \\
50 & 0.106 & 64.9 \\
60 & 0.104 & 64.7 \\
70 & 0.097 & 64.4
\end{tabular}

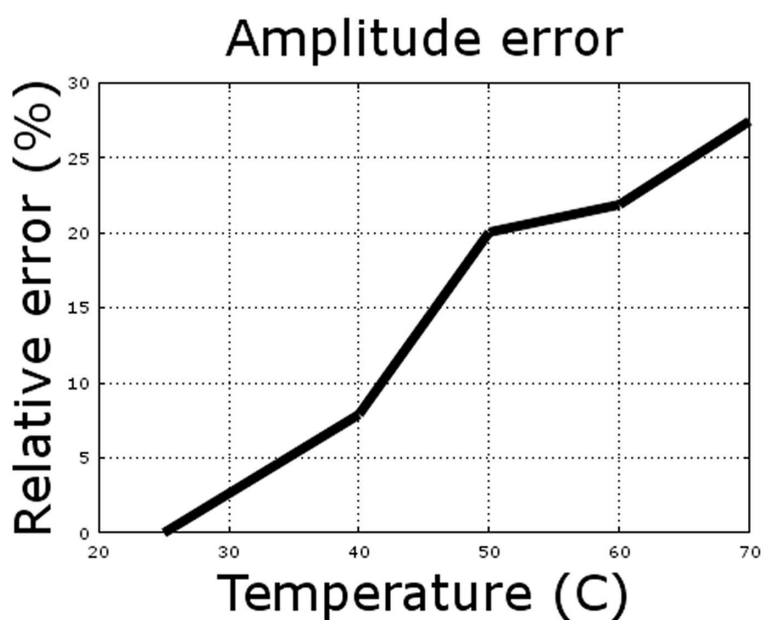

Fig. 4. Relative amplitude error in function of temperature.

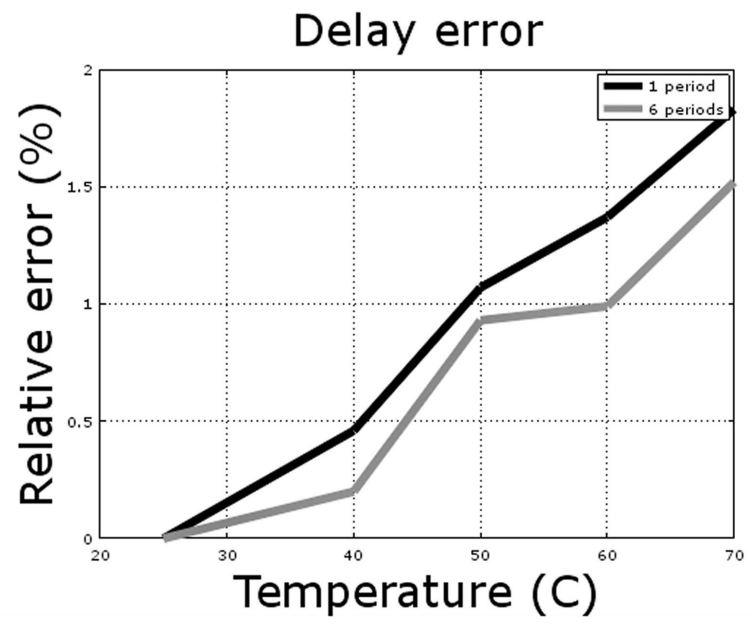

Fig. 5. Relative delay error in function of temperature.

\section{Conclusions}

The main intention of this research was the necessity to verify the influence of temperature on the properties of measurement of the magnetostrictive delay line using a tape made of amorphous alloys. 
The influence of temperature on the properties of the magnetostrictive delay line show a change of the wave amplitude of up to $27 \%$ and on the wave delay of up to $2 \%$, for temperature change from $25^{\circ} \mathrm{C}$ to $70^{\circ} \mathrm{C}$. Such a large change in the amplitude of magnetostrictive impulse can significantly affect the operation of the device.

These results should be taken into account due to their impact on the accuracy of measurements in devices using magnetostrictive delay line.

Further research concerning change in signal transition time in magnetostrictive delay line for extended temperature range and for other amorphous alloys should be conducted.

\section{Acknowledgments}

This work was partially supported by the statutory funds of Institute of Metrology and Biomedical Engineering, Warsaw University of Technology (Poland).

\section{References}

[1] E. Hristoforou, Sensor. Actuat. A Phys. 81, 142 (2000).

[2] A. Bienkowski, R. Szewczyk, J. Salach, A. Phys. Pol. A 118, 1008 (2010).

[3] R. Szewczyk, J. Salach, A. Bienkowski, M. Kostecki, A. Olszyna, A. Kolano-Burian, Solid State Phenom. 154, 29 (2009).

[4] M.R. Islam, Y. Guo, J. Zhu, in: Power Converters for Medium Voltage Networks, Springer, 2014. 